\title{
Review of Various SEO Techniques for Websites
}

\author{
Manraj Singh \\ MCA, Assistant Professor \\ PCTE Baddowal \\ Ludhiana,Punjab,India
}

\begin{abstract}
Advancement of e-commerce which includes buying and selling of products or services over the Internet has revolutionized the way a Business is done. Now consumers are becoming more comfortable buying and selling products over Internet rather than in a physical market(s). This new trend has started a cut throat competition among the websites of various business owners.

There was a time when every businessman required a descent looking website and that's it, nothing else was required. But now things have changed. Now a businessman not only requires a good looking user friendly website, but also needs to get Website(s) optimized for search engines also. This is a review paper on the various SEO Techniques that can be used by webmasters to improve website's visibility in search results and improve traffic to the website.
\end{abstract}

\section{Keywords}

On-page, SEO, Outshine, Off Page, Meta, Inbound, Outbound

\section{INTRODUCTION}

It can be termed as sum total of all techniques that are applied to bring your Website or a Webpage among the top results for a specific keyword search on a search engine.

Or in simple terms it can be defined as "Improving Visibility" of a website or a webpage in a search engine's organic (un-paid) search results for a particular keyword.

In both these definitions one term is common i.e KEYWORDwhich is some specific word or combination of words that people search for while surfing on INTERNET for eg: Buy shoes online, Book movie tickets, Best holiday destination etc. Making your website appear among the top results for a specific keyword search is what we know as Search Engine Optimization.

\section{Why SEO is required?}

Unless your website outshines all other competitor websites in search results your website will not receive enough traffic or business. There can be some websites which have poor quality content but which are better optimized for the search engine(s). These poor quality websites can have good standing in the search results in a search engine if optimized properly. So Search Engine Optimization is required for every website.

\section{An example will help you understand it better-}

Suppose a Person A has an e-commerce website selling shoes online, but there hundreds of other e-commerce websites selling the same stuff. What all techniques and changes the Person A should apply on the website in order to stay ahead of its competitors i.e Website of Person A appears as the top result when anybody searches a specific keyword.

\author{
Harmanjit Singh \\ MCA, Assistant Professor \\ PCTE Baddowal \\ Ludhiana,Punjab,India
}

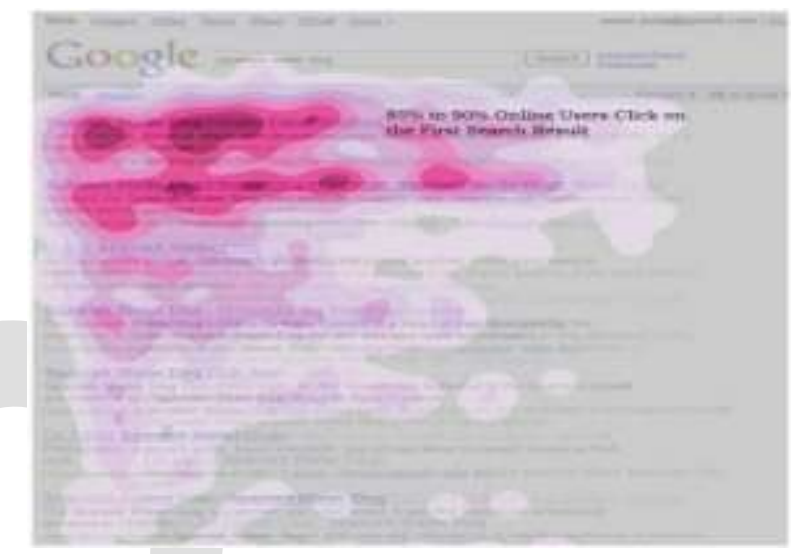

\section{How is it done?}

The most important aspect of Search Engine Optimization is making a website easy to understand and traverse for both users and search engine robots.

With advancement in technology although search engines have become increasingly smart, but still in many ways they can't read and understand a web page the same way a human does.

SEO helps a search engine figure out what exactly each webpage is about, and how important will it be for users.

\section{Broadly SEO techniques can be classified into two categories:}

1. On-page Optimization

2. Off-page Optimization

\section{On-Page Optimization:}

Any changes or modifications that are done on the webpage itself come under On-Page Optimization Techniques.

Starting from selecting the right kind of keywords, writing Meta Title, Meta Description, Meta keywords and website content containing the correct keyword density. All these come under On-Page Optimization.

\section{Step 1: (Keyword Analysis) Selecting the right keyword that suits your website.}

1. Selecting the right kind of keyword(s) for a website can be termed as the most crucial and important phase of SEO. Unless right kind of keywords are selected all other SEO techniques that will follow it, will fail. Keyword Analysis Phase forms the base of all other SEO phases that follow. It is rightly said that "Right keywords can make or break your website".

Following Research Sources can provide knowledge about keyword demand and keyword referrals.

- Google Adwords' Keyword Tool 
- Google Insights for Search

- Google Trends Keyword Demand Prediction

These online tools suggests keywords and provides estimated search volume.

What the user is searching?

- $\mathrm{MBA}$

- $\quad$ Best MBA

- Best MBA College

- Best MBA College India

- Best MBA College in World

\section{Google Adword Keyword Tool}

\section{Find keywords}

Based on one or both of the following:

\begin{tabular}{|c|c|c|}
\hline \multicolumn{2}{|l|}{ Word or phrase (one per line) } & Website \\
\hline best mba colege in world & & \\
\hline & & 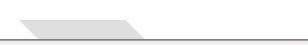 \\
\hline Keppond & Conpstion & Gictel Innthy Searches 3 \\
\hline to best novastous & E & 5,000 \\
\hline A dost roverogens & E & 14,000 \\
\hline tó rtacrlegein & E & 166,000 \\
\hline 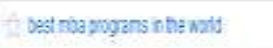 & E & 1,000 \\
\hline to bestoleges nta natt & $\square$ & 14,800 \\
\hline ff meacologen in usz & EI & 1900 \\
\hline tost nova unetst & E & 2900 \\
\hline 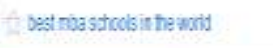 & EI & 1900 \\
\hline tost nuversas trata & I & 14,800 \\
\hline A dost nibarals & DI & 4,000 \\
\hline thost noacley: & E & 18,100 \\
\hline A dost nuavine & E & 8,000 \\
\hline th rte bestodsy: & E & 5,000 \\
\hline Acost doleges inusa & II & 14,800 \\
\hline thost nuadayes & E & 12,000 \\
\hline A dost bcodie mba & EI & 2900 \\
\hline 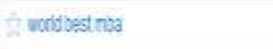 & E & 4,000 \\
\hline 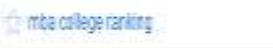 & III & 8,900 \\
\hline tha nogabsys nwerd & in & 4,400 \\
\hline Af Jost nogia usa & E & 1900 \\
\hline Licologes & 目 & $T 480,000$ \\
\hline
\end{tabular}

Even the pictures which are posted online required a suitable name, so that it is easier for the Search Engines to understand a photograph. Fortunately, SEO allows webmasters to provide "clues" that the engines can use to understand content. In fact, adding proper structure to your content is essential to SEO.

Understanding both the abilities and limitations of search engines allows you to properly build, format and annotate your web content in a way that search spiders can digest. Without SEO, many websites remain invisible to search engines.

\section{Step 2: Writing Meta Title, Meta Description and Meta Keywords}

There are certain specific rules that need to be followed while writing Meta Title, Meta Description and Meta Keywords for each and every page.

Length of Title should be less than 65 characters.

Always place the most important keywords near the beginning of the page title.

Target the most important competitive keyword phrase at the home page.

Target one or two keywords phrases per page.

Put some main keyword in Keyword Meta Tag.

Write a good description for Meta Description tag with 3-4 keywords

Description must be unique to each page.

Keep Meta description short and meaningful write only 1 or 2 sentences in description. Not to exceed 240 characters

\section{Step 3: Inbound and Outbound Links}

Inbound links: Improving Inbound links through Link Building:

Webmasters of similar websites can mutually exchange their links to improve Inbound links to their websites. For eg webmaster of xyz.com website can put a link of abc.com website on its homepage and webmaster of abc.com website can do vice-versa. More the number of Inbound links, the better it gets. Exchange of links among websites with same page rank is beneficial for both the parties.

\section{Outbound Links:}

Not only Inbound inks but Outbound links are also as important and beneficial for a website. Suppose while writing some content a content writer finds a good webpage on that particular topic. Creating a hyperlink in your content to that particular source will only benefit your customer or reader and hence creating Outbound Links are strongly recommended. Even an Outbound link to your own particular webpage will improve your website navigation and its helps in SEO of a website.

Step 4: Writing Quality Content

\section{Optimizing Images}


Content is the KING. Writing quality and unique content is the most important task that can do wonders for a website. No matter how much money, time and energy is spent on optimizing a website, unless it has unique and quality content all SEO techniques are bound to Fail. Therefore it is always suggested to Never Copy Content from any online source.

While writing any content following points should be taken into consideration:

1. Place the most important keyword in $\mathrm{H} 1$ tag.

2. Use $\mathrm{H} 2$ and $\mathrm{H} 3$ for sub headers where required.

3. Use Bold / Italic / Underline for your keyword phrase for extra weight in contents.

4. Use bulleted lists to make content easier to read.

5. Use ALT tag for images, so that crawler can know about images.

6. Use text based navigation.

7. Use videos on your site which is related to your niche.

8. Write informative, fresh, unique, useful content on your site.

9. Write site content in between 300 to 500 words.

10. Keywords $\%$ must be 3 to $5 \%$.

11. Don't copy any content from other websites, fresh and unique content is the key of your success.

12. Regular update your website with fresh content.

13. Write your content for human not for robot.

14. Use keywords rich URL instead of dynamic URL.

15. Break your article in paragraph if your article is long.

\section{Off- Page Optimization}

Off-Page Optimization can be termed as a set of things done on other website(s) that drive traffic to your website. Off-Page Optimization majorly includes submitting details of your website to Directories, Bookmarking Websites and Social Networking Websites etc. People often spend too much time and energy on On-Page Optimization, working on things like keyword density and keyword stuffing, and not enough time off-page. But the bottom line is, it is the off-page that gets you traffic, that gets you rankings.

Well, the first thing to understand is that the primary focus of off-page optimization is creating Inbound Links/Backlinks to your web site. The search engines assume that if you have more backlinks to your website, Than your web site is more 'popular' than other similar web sites. Now, there is an issue of quality with the backlinks - If your web page is getting single backlink from a web page with a descent Page Rank, it will be more useful than 10 backlinks from web pages with zero Page Rank.

So backlinks only from quality web sites is given due importance by the search engines while displaying search results.

So here are the basics:

1) Sitemap Submission:- A site map (or sitemap) is a list of pages of a web site accessible to crawlers or users. Submit Sitemap of your Website Google Webmaster Tools and get Google's view of your site and diagnose problems See how Google crawls and indexes your site and learn about specific problems having accessing it. Whenever some new pages are added to the website, try submitting a fresh SiteMap to Google and Other Search Engines.
2) Article Submission: Only writing Articles with good good keyword density is not enough, they must be submitted to the highest ranked Article Directories Online. These Articles must be unique and should have 2-3 backlinks to specific pages on your web site. This helps improve readership and link popularity of your website.

3) Directory Submission: There are some directory websites online which store the list of websites category wise. For eg Entertainment Websites, Lawyers, Doctors. A webmaster must submit web site's URL to the top ranked web directories online for eg Demoz, this also help improve the back link numbers.

4) Bookmarking: Recently a new trend has started in which people share quality and interesting Post/Articles on their profile pages on Social Networking websites such as Facebook. Webmasters must submit Posts/Articles on some Social Networking Websites and specific Bookmarking Websites such as Digg, Delicious, Diigo etc.

\section{Do's and Dont's of SEO}

- Create Quality Content, without it all SEO techniques will fail.

- $\quad$ Proper mix of Keywords (Keyword Density) should be used

- Write content for users not for search engines. It will help you keep your bounce rate low.

- Don't be obsessed with PageRank. A site with lower PR can actually outrank one with a higher PR.

- If your site content doesn't change often, your site needs a blog because search spiders like fresh text. Blog at least three time a week

- Verify your website in google webmaster tool

- When link building, think quality, not quantity. One single, good, authoritative link can do a lot more for you than a dozen poor quality links

- $\quad$ Spend time on your competitor's websites and their target keywords

- Check the content, title and meta of your competitor's website.

\section{References:}

1. Always Be Testing: The Complete Guide to Google Website Optimizer - Bryan Eisenberg \& John Quarto-von Tivadar

2. The Art of SEO - Eric Enge, Jessie Stricchiola, Rand Fishkin and Stephan Spencer

3. The Truth about Search Engine Optimization Rebecca Lieb

4. Inbound Marketing - Brian Halligan \& Dharmesh Shah 